(C) 2015 IEEE. Personal use of this material is permitted. Permission from IEEE must be obtained for all other uses, in any current or future media, including reprinting/republishing this material for advertising or promotional purposes, creating new collective works, for resale or redistribution to servers or lists, or reuse of any copyrighted component of this work in other works. 


\title{
Predictive Optimal Switching Sequence Direct Power Control for Grid Connected Power Converters
}

\author{
Sergio Vazquez, Member, IEEE, Abraham Marquez, Ricardo Aguilera, Member, IEEE, Daniel Quevedo, Senior \\ Member, IEEE, Jose I. Leon, Senior Member, IEEE and Leopoldo G. Franquelo, Fellow, IEEE
}

\begin{abstract}
Grid connected power converters play a key role in several applications such as integration of renewable energy sources and motor drives. For this reason, the development of high performance control strategies for this particular class of power converters has increasingly attracted the interest of both academic and industry researchers. This paper presents the predictive Optimal Switching Sequence Direct Power Control (OSS-DPC) algorithm for grid connected converters. The OSSDPC method belongs to the predictive direct power control (P-DPC) family and provides the desired power references by calculating globally optimal switching sequences. To address computational and implementation issues, an efficient control algorithm, named reduced OSS-DPC (ROSS-DPC) is introduced. The implementation of the proposed control strategy in a standard DSP is evaluated on a two-level power converter prototype working as a STATCOM. Experimental results show algorithm's potential to provide high performance during both transient and steady states.
\end{abstract}

\section{INTRODUCTION}

G RID connected power converters play a key role in many industrial applications such as active front end (AFE) converters for motor drives, integration of renewable energy sources (wind or photovoltaic), and energy storage systems [1]-[4]. To achieve high performance, several issues have been studied in the literature, including converter topologies, modulation strategies, grid synchronization schemes, and control algorithms [5]-[10].

The control scheme of a grid connected converter is usually divided into two control loops. The external one is devoted to regulate the dc-link capacitor voltage. The inner control loop focuses on either tracking the current or instantaneous active and reactive power references depending on the state variables

Manuscript received January 29, 2014; revised March 25, 2014, May 9 , 2014 and July 29, 2014; accepted July 29, 2014.

Copyright (C) 2014 IEEE. Personal use of this material is permitted. However, permission to use this material for any other purposes must be obtained from the IEEE by sending a request to pubs-permissions@ieee.org.

This work was supported by the Ministerio Español de Economia y Competitividad under project ENE2012-36897 and by the Consejeria de Innovación Ciencia y Empresa (Junta de Andalucia) under the project P11TIC-7070

S. Vazquez, A. Marquez, J.I Leon and L.G. Franquelo are with the Electronic Engineering Department, Universidad de Sevilla (Spain), (e-mail: sergi@us.es).

Ricardo Aguilera is with the Australian Energy Research Institute, The University of New South Wales, Australia (raguilera@ieee.org).

Daniel Quevedo is with the School of Electrical Engineering and Computer Science, The University of Newcastle, Australia (dquevedo@ieee.org). used to design the control strategy. In both cases, it is possible to use an indirect control approach by including a PWM-SVM modulation scheme [11], [12].

The direct power control (DPC) method uses the instantaneous active and reactive powers as state variables. In its conventional form, DPC directly selects the power switch states to track the desired active and reactive powers [13]. The main problem with this approach is that the resulting switching frequency is variable. This complicates the design of the output filter. To overcome this issue, DPC formulated as an indirect control was proposed in [14]. In order to avoid performance degradation by the mismatching of system parameters, an adaptive DPC control law has also been developed [15].

Although [14], [15] perform well, two major issues remain. First, the method to define the control design constants is not always clear and usually requires a trial and error procedure. Second, conventional PI controllers or integral structures are used to derive the control action. These factors limit the bandwidth of the DPC algorithm, sacrificing the dynamic response of the system.

As an alternative to these approaches, model predictive control (MPC) has been applied to the design of DPC strategies (MPC-DPC). In general, MPC solves, at each sampling instant, an optimal control problem over a finite prediction horizon. This optimization yields an optimal control sequence for the whole prediction horizon. Following the receding horizon principle, only the first control action of this optimal sequence is applied to the system. This process is repeated at each sampling instant using fresh state measurements or estimates [16]-[19].

Depending on the nature of the input constraints, MPC can be divided into two major groups. If the power converter uses a modulator then the input will be constrained to belong to a bounded continuous control set (e.g., duty cycles or PWM references). In the case when the converter switches are handled directly, the input will belong to a Finite Control Set (FCS) [18]. In the power electronics literature, both continuous and FCS-MPC algorithms have been proposed for the DPC of power converters [20]-[23]. To highlight this difference, in the present work they will be referred to as P-DPC and FCS-PDPC respectively.

FCS-P-DPC considers the converter switches as the system input. A power cost function, which comprises future active and reactive power tracking errors is evaluated for each 
possible switch combination. The optimal input to be applied to the system is the one that minimizes this cost function. FCS-P-DPC is able to provide good performance. However, it requires high sampling frequency and provides a variable switching frequency. This generates output currents with a widespread harmonic spectrum [20], [21].

Alternatively, P-DPC considers the commutation instants $t_{i}$, within a sampling period $T_{s}$, as the system input. To do this, for each sampling period, P-DPC selects a converter voltage vector sequence from a table using the grid voltage vector angle. Thus, the minimization of the power cost function returns the optimal commutation instants $t_{i}^{o p}$ for these vectors within the sampling period. This generates a switching pattern that provides a constant switching frequency [24]. The robustness of this strategy can be improved by using observers to identify uncertain system parameters such as the smoothing inductor value [25], [26]. Conventional P-DPC achieves, in general, good closed-loop behavior. Nonetheless, at times, its performance will be affected by an incorrect voltage sequence selection [27]. To solve this problem, revised tables defining the optimal output voltage sequence have been proposed in [28]. In essence, when a switching time becomes negative, then a new voltage sequence is selected. Although this procedure presents good results, it requires additional computations. To overcome this issue, a P-DPC strategy, which requires neither grid voltage sector information nor a voltage vector selection process, was proposed in [29]. In this strategy, the controller simply evaluates the switching times for one sector and, from this information, reconstructs the optimal switching pattern to be applied.

The associated constrained optimization problem has also been addressed in [30]. It should be noted that natural constraints for this problem are the switching period and the magnitude of the control voltage vector. The solution to the constrained problem proposed in [31], consists of calculating the global optimum from the quadratic cost function of the instantaneous power deviations at the end of the predicted switching instant. Thus, the control vector is chosen as the orthogonal projection of the global optimum to the hexagon of the control vectors.

The present paper introduces a novel P-DPC strategy. To overcome the aforementioned problems inherent to voltage sequence selection, the use of look-up-tables is avoided. Instead, the proposed P-DPC takes advantage of the discrete nature of the power converter. As is well known, the control region of the power converter in the stationary $\alpha \beta$ frame can be divided into six sectors. Taking this into account, the proposed controller calculates the optimal commutation instants for each sector. This provides six local Optimal Switching Sequences (OSSs) and their associated cost values. Similar to the FCSMPC approach, the global OSS applied by the proposed PDPC is that which minimizes the power cost function. The main advantage of the proposed predictive controller when compared to P-DPC is that the control action is calculated without relying on the grid voltage sector information. This allows the controller to select the OSS even when the control action is saturated. The proposed predictive control strategy is called OSS-DPC.

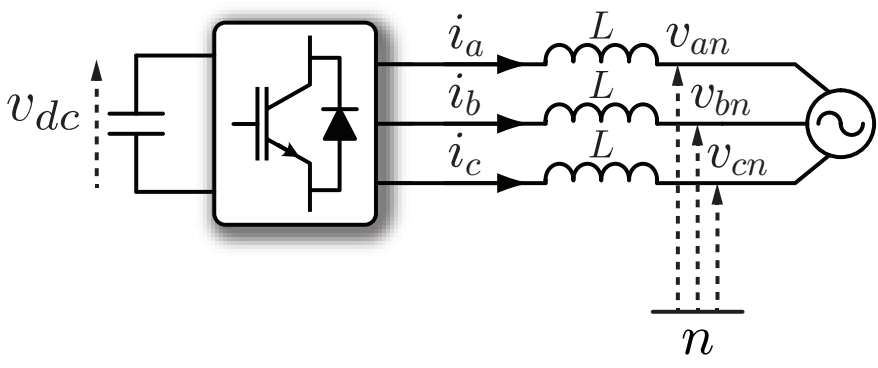

Fig. 1. Scheme of a grid connected two-level power converter.

The present article extends the preliminary work presented in [32]. In the latter, only a simulation study was carried out. In the present work, the proposed OSS-DPC is experimentally tested in order to evaluate the effectiveness of the proposed strategy when implemented on a standard DSP platform. It turns out that OSS-DPC requires a large number of computations. To address this issue, a new algorithm, named Reduced OSS-DPC (ROSS-DPC) is developed. ROSS-DPC presents a reduced computational cost when compared to OSS-DPC, allowing one to take advantage of the digital platform to improve the closed-loop performance. In addition, in this paper the effect of delays caused by the digital implementation are also investigated and a delay compensator is introduced.

The remainder of this paper is organized as follows. In Section II the conventional P-DPC controller is described. In Section III the proposed OSS-DPC is derived. Experimental results are documented in Section IV. Here, in order to address practical experimental issues, the ROSS-DPC algorithm is introduced. Conclusions are given in Section V.

\section{COnVEntional P-DPC ALGORithm}

The key idea of the conventional P-DPC strategy is to use a predefined switching pattern based on the available switching vectors that the converter can generate. In order to minimize the active and reactive power errors, the method finds, in an optimal manner, the instants when these vectors are applied within a given sampling period. For this purpose, this section develops a system model which is then used to analyze the instantaneous active and reactive power dynamics [22], [24].

\section{A. System Model}

Fig. 1 shows the electrical scheme of a voltage source inverter (VSI) connected to the grid. Considering the stationary $\alpha \beta$ framework, the instantaneous active and reactive power, $p_{s}$ and $q_{s}$, can be expressed by [33]:

$$
\left[\begin{array}{c}
p_{s} \\
q_{s}
\end{array}\right]=\left[\begin{array}{cc}
v_{s \alpha} & v_{s \beta} \\
v_{s \beta} & -v_{s \alpha}
\end{array}\right]\left[\begin{array}{c}
i_{s \alpha} \\
i_{s \beta}
\end{array}\right]
$$

where $v_{s \alpha \beta}$ and $i_{s \alpha \beta}$ represent the grid voltage and the VSI output current in the $\alpha \beta$ frame respectively by using the 


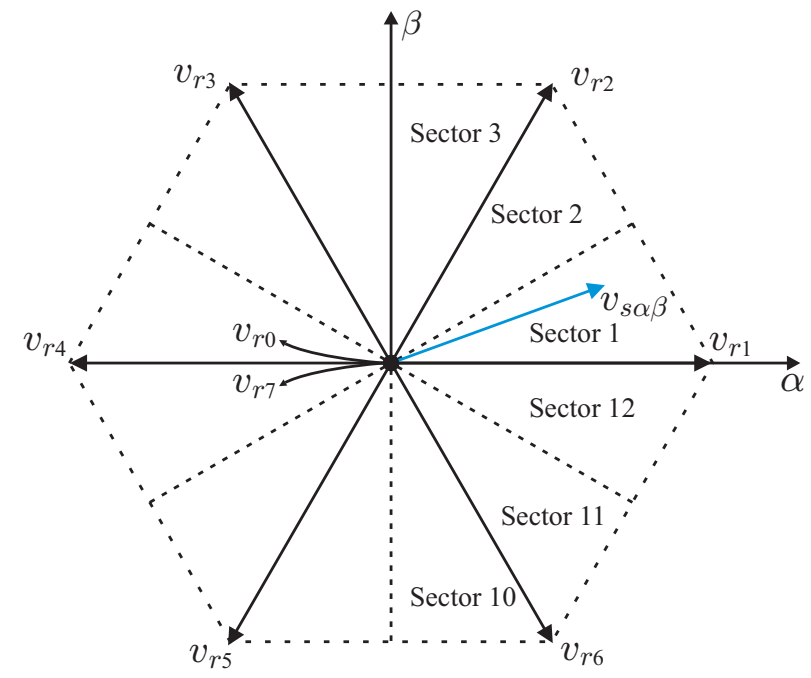

Fig. 2. Converter voltage vectors $v_{r i}$.

power-invariant Clarke transformation. From (1), it is possible to derive dynamic equations for $p_{s}$ and $q_{s}$ as

$$
\begin{aligned}
& \frac{d p_{s}}{d t}=v_{s \alpha} \frac{d i_{s \alpha}}{d t}+v_{s \beta} \frac{d i_{s \beta}}{d t}+i_{s \alpha} \frac{d v_{s \alpha}}{d t}+i_{s \beta} \frac{d v_{s \beta}}{d t}, \\
& \frac{d q_{s}}{d t}=v_{s \beta} \frac{d i_{s \alpha}}{d t}-v_{s \alpha} \frac{d i_{s \beta}}{d t}-i_{s \beta} \frac{d v_{s \alpha}}{d t}+i_{s \alpha} \frac{d v_{s \beta}}{d t} .
\end{aligned}
$$

Under a balanced sinusoidal three-phase system, the derivative of the grid voltage can be expressed as

$$
\frac{d v_{s \alpha \beta}}{d t}=\omega J v_{s \alpha \beta},
$$

where the matrix $J$ is given by

$$
J=\left[\begin{array}{rr}
0 & -1 \\
1 & 0
\end{array}\right] .
$$

Taking into account that the dynamic model of the ac currents can be represented by

$$
\frac{d i_{s \alpha \beta}}{d t}=\frac{1}{L}\left(v_{r \alpha \beta}-v_{s \alpha \beta}\right),
$$

where $v_{r \alpha \beta}$ is the output voltage of the VSI, the following dynamic model of the active and reactive powers is obtained

$$
\begin{aligned}
\frac{d p_{s}}{d t} & =v_{s \alpha}\left(\frac{1}{L}\left(v_{r \alpha}-v_{s \alpha}\right)+\omega i_{s \beta}\right) \\
& +v_{s \beta}\left(\frac{1}{L}\left(v_{r \beta}-v_{s \beta}\right)-\omega i_{s \alpha}\right), \\
\frac{d q_{s}}{d t} & =v_{s \beta}\left(\frac{1}{L}\left(v_{r \alpha}-v_{s \alpha}\right)+\omega i_{s \beta}\right) \\
& -v_{s \alpha}\left(\frac{1}{L}\left(v_{r \beta}-v_{s \beta}\right)-\omega i_{s \alpha}\right) .
\end{aligned}
$$

\section{B. Vector Selection}

As illustrated in Fig. 2 a two-level power converter can generate eight voltage vectors, $v_{r i}$ with $i \in\{0, \ldots, 7\}$ in the $\alpha \beta$ plane. In the conventional P-DPC [22], the vector sequence applied to the converter is selected in order to
TABLE I

VECTOR SEQUENCE TO BE APPLIED WHEN USING CONVENTIONAL P-DPC

\begin{tabular}{|c|c|}
\hline Sector & Vector sequence $\vec{v}_{j}$ \\
\hline 1 & 127721 \\
2 & 012210 \\
3 & 032230 \\
4 & 327723 \\
5 & 347743 \\
6 & 034430 \\
7 & 054450 \\
8 & 547745 \\
9 & 567765 \\
10 & 056650 \\
11 & 016610 \\
12 & 167761 \\
\hline
\end{tabular}

minimize the switching losses. To do this, the $\alpha \beta$ plane is divided into twelve sectors as depicted in Fig. 2. Each sector is assigned to a vector sequence, $\vec{v}_{j}=\left\{v_{1}, v_{2}, v_{3}, v_{3}, v_{2}, v_{1}\right\}$ with $j \in\{1, \ldots, 12\}$ as presented in Table I. The sequence to be applied to the converter during the sampling period, $T_{s}$, is chosen depending on the sector in which the grid voltage lies. For example, in Fig. 2, $v_{s \alpha \beta}$ lies inside sector 1, thus the sequence to be applied is $\vec{v}_{1}=\left\{v_{r 1}, v_{r 2}, v_{r 7}, v_{r 7}, v_{r 2}, v_{r 1}\right\}$.

\section{Application Times}

Once the switching pattern is selected, the commutation instants $t_{1}, t_{2}$, and $t_{3}$ for the three voltage vectors $v_{i}$ with $i \in\{1,2,3\}$ that form the switching sequence $\vec{v}_{j}$ need to be calculated. The P-DPC approach adopted in [22] is based on the dynamic model presented in (6). To be more specific, the predictions for the active and reactive powers at instant $k+1$ are calculated as

$$
\begin{aligned}
& p_{s, k+1}=p_{s, 0}+2 \sum_{i=1}^{3} f_{p i} t_{i} \\
& q_{s, k+1}=q_{s, 0}+2 \sum_{i=1}^{3} f_{q i} t_{i},
\end{aligned}
$$

where $p_{s, k+1}$ and $q_{s, k+1}$ are the predictions for instant $k+1$, $p_{s, 0}$ and $q_{s, 0}$ are the initial values of $p_{s}$ and $q_{s}$ at instant $k$, $f_{p i}, f_{q i}$ are the increments for the instantaneous active and reactive powers, and $t_{i}$ are the application times of vectors $v_{r i}$. If it is assumed that $f_{p i}$ and $f_{q i}$ are constant during the sampling interval, then $f_{p i}=f_{p i, k}$ and $f_{p i}=f_{q i, k}$, where $f_{p i, k}$ and $f_{q i, k}$ are the values at instant $k$ for the vector $v_{i}$. These can be calculated from (6) as

$$
\begin{gathered}
f_{p i, k}=\left.\frac{d p_{s}}{d t}\right|_{v_{r}=v_{i}, v_{s}=v_{s, k}, i_{s}=i_{s, k}} \\
f_{q i, k}=\left.\frac{d q_{s}}{d t}\right|_{v_{r}=v_{i}, v_{s}=v_{s, k}, i_{s}=i_{s, k}}
\end{gathered} .
$$

To obtain the commutation instants $t_{i}$, the conventional PDPC minimizes the quadratic function

$$
J\left(p_{s, k+1}, q_{s, k+1}\right)=e_{p, k+1}^{2}+e_{q, k+1}^{2}
$$




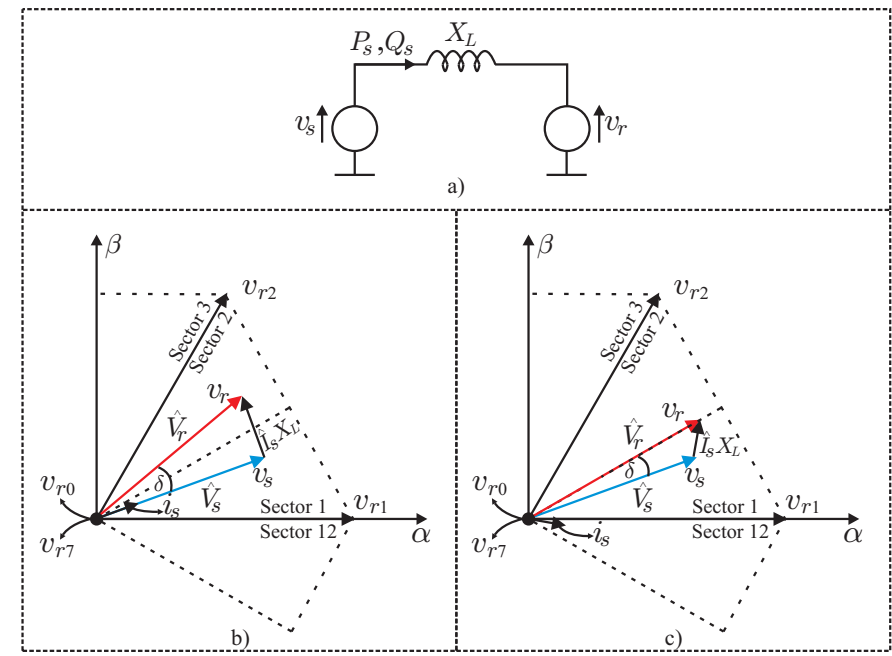

Fig. 3. Vectorial representation of the steady state. a) Simplified system. b) Required inverter voltage. c) Constrained inverter voltage using conventional P-DPC

with $e_{p, k+1}=p_{s}^{*}-p_{s, k+1}$ and $e_{q, k+1}=q_{s}^{*}-q_{s, k+1}$, where $p_{s}^{*}$ and $q_{s}^{*}$ are the reference values for the instantaneous active and reactive power respectively.

In [22], an explicit solution to the above problem is found by considering an unconstrained problem. This yields the following application times

$t_{1}=\frac{\left(f_{q 2}-f_{q 3}\right) e_{p k}+\left(f_{p 3}-f_{p 2}\right) e_{q k}+\left(f_{p 2} f_{q 3}-f_{p 3} f_{q 2}\right) T_{s}}{2\left(\left(f_{q 2}-f_{q 3}\right) f_{p 1}+\left(f_{q 3}-f_{q 1}\right) f_{p 2}+\left(f_{q 1}-f_{q 2}\right) f_{p 3}\right)}$,

$t_{2}=\frac{\left(f_{q 3}-f_{q 1}\right) e_{p k}+\left(f_{p 1}-f_{p 3}\right) e_{q k}+\left(f_{q 1} f_{p 3}-f_{q 3} f_{p 1}\right) T_{s}}{2\left(\left(f_{q 2}-f_{q 3}\right) f_{p 1}+\left(f_{q 3}-f_{q 1}\right) f_{p 2}+\left(f_{q 1}-f_{q 2}\right) f_{p 3}\right)}$,

$t_{3}=\frac{T_{s}}{2}-t_{1}-t_{2}$

where $e_{p, k}=p_{s}^{*}-p_{s, 0}, e_{q, k}=q_{s}^{*}-q_{s, 0}$ and $T_{s}$ represents the sampling period.

\section{OSS-DPC TECHNIQUE}

Conventional P-DPC, as shown in Section II, constitutes a simple strategy that often provides good performance when controlling grid connected power converters. However, at times, the voltage sequence selection method used by $\mathrm{P}$ DPC will perform poorly [27]. The vectorial representation presented in Fig. 3 illustrates this problem. In essence, $v_{r}$ should be generated using the voltage vectors belonging to the sector where $v_{s}$ is located. Then, at times, it is not possible to generate the required voltage to achieve the instantaneous active and reactive power references [32].

To overcome this problem, [32] proposed an algorithm to optimally obtain the sector in which the inverter voltage, $v_{r}$, will be synthesized. The key idea of this method, named OSSDPC, is to calculate the local optimal times in (10), for every sector, i.e., $T_{j}=\left[t_{1}, t_{2}, t_{3}\right]$ for all $j \in\{1, \ldots, 12\}$. These times are used to evaluate, in each sector $j$, the cost function

$$
J_{j}\left(t_{1}, t_{2}, t_{3}\right) \triangleq \sum_{i=1}^{i=6}\left(p_{s}^{*}-p_{s i}\right)^{2}+\left(q_{s}^{*}-q_{s i}\right)^{2}
$$

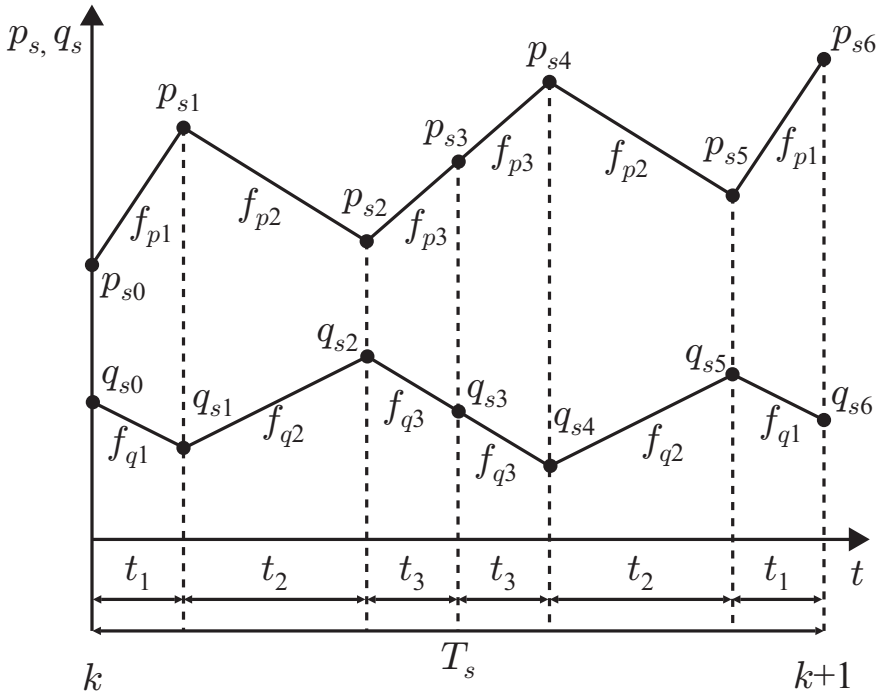

Fig. 4. Active and reactive power trajectories during one sampling period.

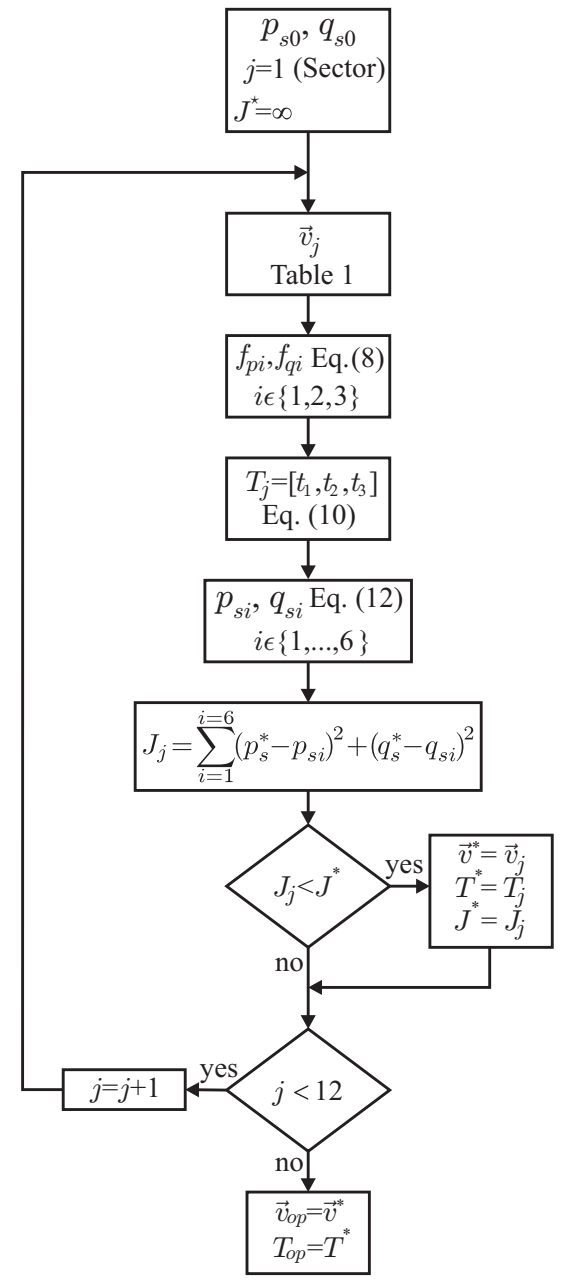

Fig. 5. Flow diagram of the proposed OSS-DPC strategy.

where $p_{s i}$ and $q_{s i}$ are defined as

$$
\begin{aligned}
p_{s i} & =p_{s(i-1)}+f_{p i} t_{i} \\
q_{s i} & =q_{s(i-1)}+f_{q i} t_{i},
\end{aligned}
$$




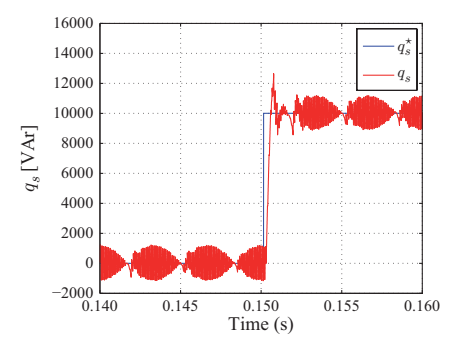

(a)

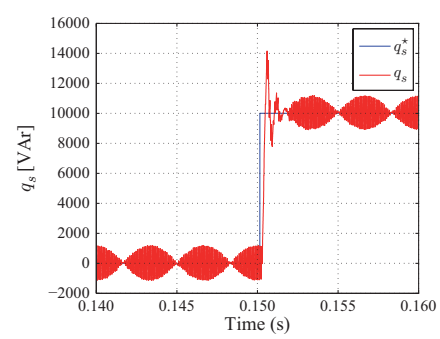

(b)
Fig. 6. System response to an instantaneous reactive power command step from $q_{s}^{*}=0 \mathrm{kVAr}$ to $q_{s}^{*}=10 \mathrm{kVAr}$ : (a) P-DPC algorithm, (b) OSS-DPC algorithm.

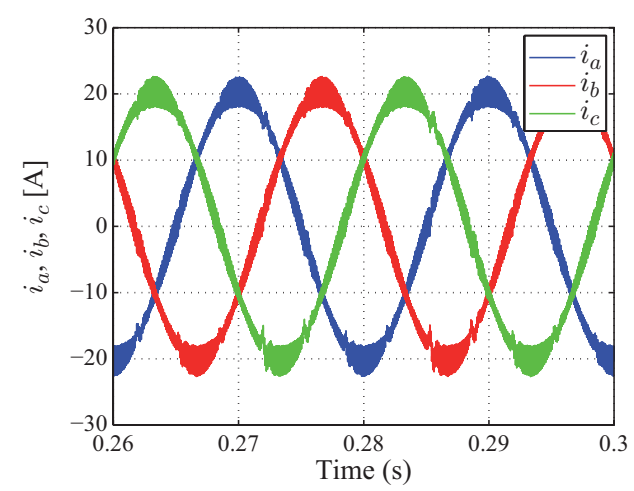

(a)

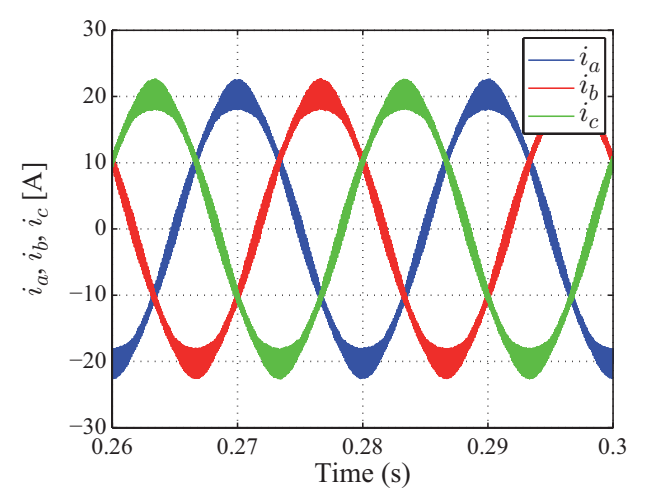

(b)

Fig. 7. Steady state grid currents for instantaneous reactive power reference $q_{s}^{*}=10$ kVAr: (a) P-DPC algorithm, (b) OSS-DPC algorithm.

with $p_{s 0}=p_{s, 0}, q_{s 0}=q_{s, 0}, t_{4}=t_{3}, t_{5}=t_{2}, t_{6}=t_{1}$, $f_{p 4}=f_{p 3}, f_{p 5}=f_{p 2}$ and $f_{p 6}=f_{p 1}$. Fig. 4 illustrates the evolution of $p_{s}$ and $q_{s}$ from instant $k$ to $k+1$

The twelve resulting cost values, $J_{j}$, are local minima. The optimal sector, $j_{o p}$, is chosen as the one which minimizes the cost function in (11). The optimal times for this sector are $T_{o p}$, whereas $\vec{v}_{o p}$ is the global optimal switching sequence. In Fig. 5, a flow diagram of the OSS-DPC algorithm is presented.

The main advantage of using (11) when compared to (9) is that it evaluates the predictions for the instantaneous active and reactive powers at six different instants within the sampling period. This improves the inter-sample behavior, cf. [34].

To show the benefits of using the OSS-DPC, a simulation has been carried out using the parameters presented in Table II. Fig. 6 compares the response to a step reference change in
TABLE II

SYSTEM PARAMETERS

\begin{tabular}{cc}
\hline \hline Parameter & Value \\
\hline Smoothing inductor & $L=2 \mathrm{mH}$ \\
DC-Link capacitor & $C=2200 \mu \mathrm{F}$ \\
Grid phase voltage & $v_{a n}, v_{b n}, v_{c n}=230 \mathrm{~V}_{r m s}$ \\
Grid frequency & $f=50 \mathrm{~Hz}$ \\
Sampling frequency & $f_{s}=10 \mathrm{kHz}$ \\
Switching frequency & $f_{s w}=10 \mathrm{kHz}$ \\
\hline \hline
\end{tabular}

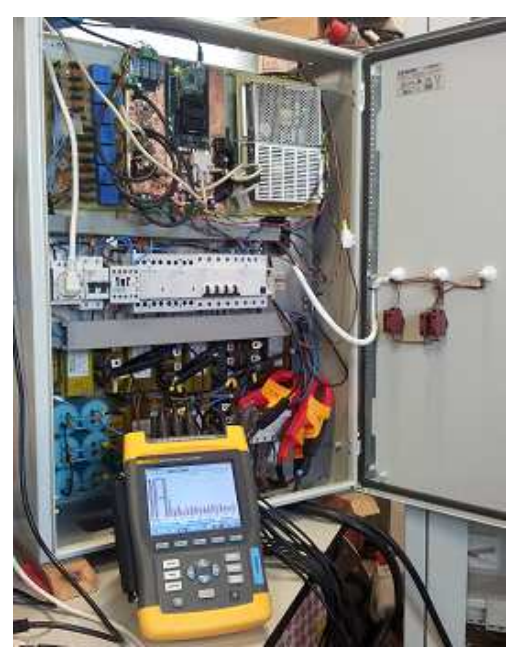

Fig. 8. Converter prototype.

$q_{s}^{*}$ using conventional P-DPC and the proposed OSS-DPC algorithms. Fig. 6a and Fig. 6b show that both strategies present similar dynamic responses, although a slightly faster response can be observed when OSS-DPC is used. However, OSS-DPC offers a clear improvement when compared with standard P-DPC in the steady state. To highlight this, Fig. 7 presents the grid current waveforms. Fig. 7a illustrates that conventional P-DPC periodically selects an incorrect switching sequence. This generates spikes in the grid currents, which increase their total harmonic distortion (THD). On the other hand, the grid currents obtained by OSS-DPC do not have spikes, as shown in Fig. 7b. This is due to the fact that OSSDPC explores all vector sectors when obtaining the optimal switching sequence.

\section{EXPERIMENTAL RESULTS}

The proposed OSS-DPC algorithm has been tested on a laboratory prototype system comprising a power converter connected to the grid. The experimental setup consists of a three-phase two-level converter working as a STATCOM. The electrical scheme of the system is shown in Fig. 1. The main system parameters are summarized in Table II.

The converter prototype is shown in Fig. 8. The core of the control hardware is based on the TMS320F28335 DSP where the OSS-DPC algorithm has been coded. During the experiments, instantaneous reactive power command steps have been introduced in order to evaluate performance.

Fig. 9 shows the transient response of the OSS-DPC when a command step is produced in the instantaneous reactive 


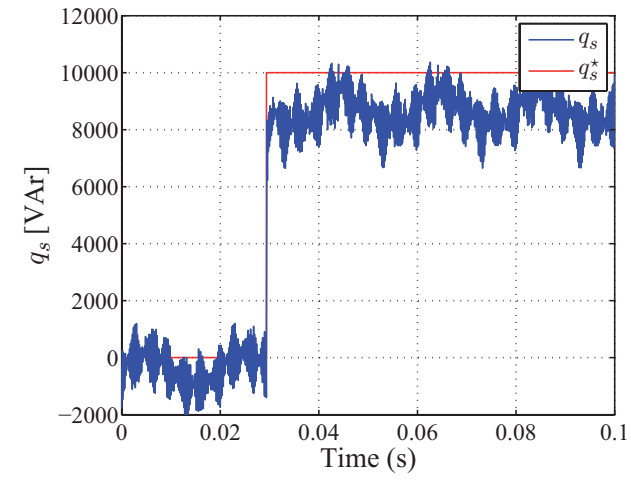

Fig. 9. System response to an instantaneous reactive power command step from $0 \mathrm{kVAr}$ to $10 \mathrm{kVAr}$

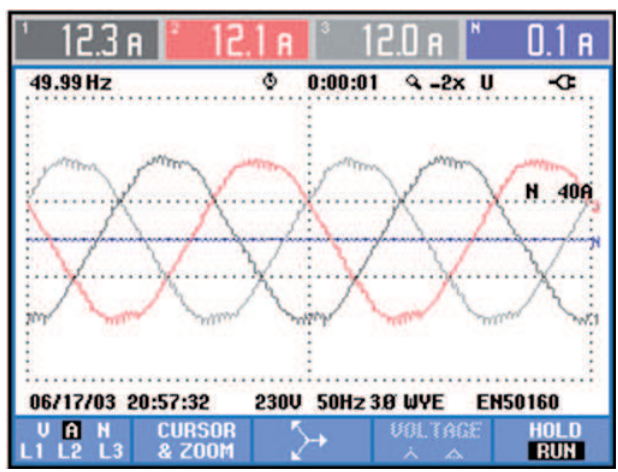

(a)

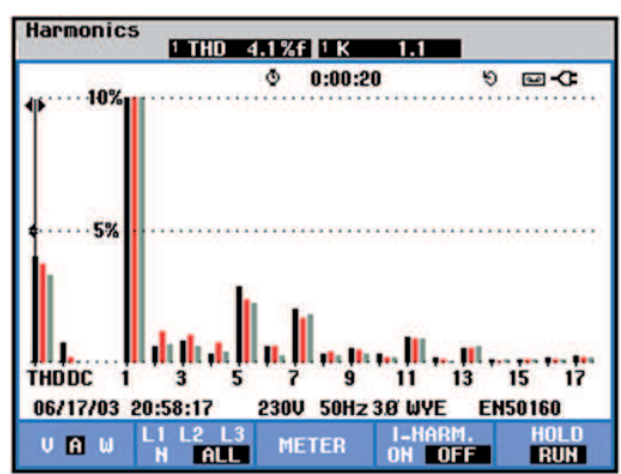

(b)

Fig. 10. Steady state output currents for an instantaneous reactive power command of 10 kVAr: (a) Output currents, (b) Harmonic content.

power from no load to $10 \mathrm{kVAr}$. The system response is very fast and the new reference is reached almost instantaneously. However, in steady state, there exists a difference between the command and actual value of $q$. This is mainly caused by model inaccuracies, and the delay introduced by the digital platform. The latter issue can be compensated in the algorithm design as will be shown later in Section IV-B. The output currents of the VSI are shown in Fig. 10. It should be noted that the low order harmonics in the output currents are due to the grid voltage in the laboratory utility, which contains $5^{\text {th }}$ and $7^{\text {th }}$ harmonics. The THD value of the output current is $4.1 \%$.

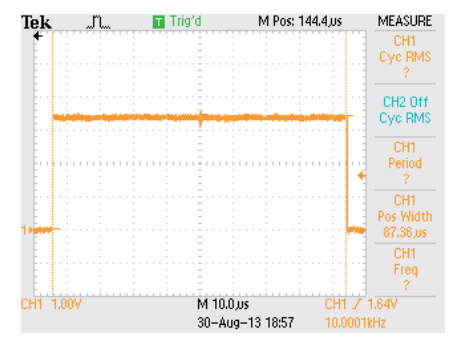

(a)

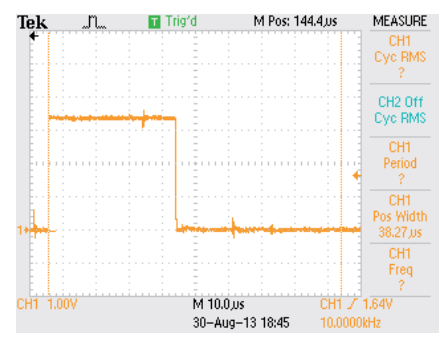

(b)
Fig. 11. Execution time of the control strategies: (a) OSS-DPC algorithm, (b) ROSS-DPC algorithm.

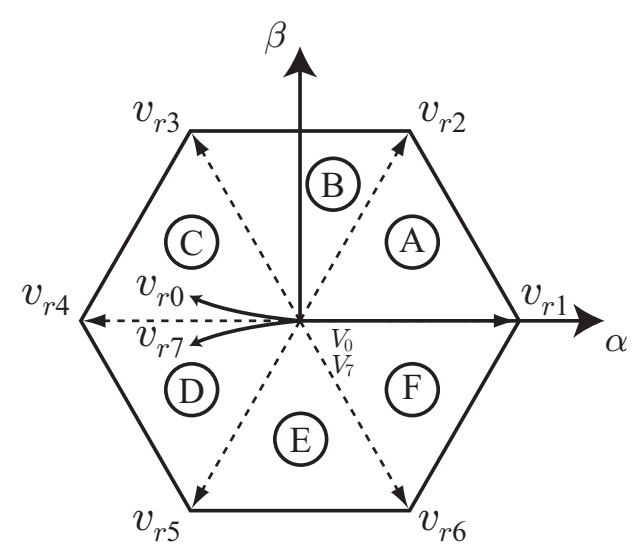

Fig. 12. Sectors considered in the ROSS-DPC algorithm.

\section{A. Analysis of the computational burden}

One of the major concerns about MPC algorithms is the computational cost. In general, MPC strategies rely upon significant online computations and therefore require powerful hardware platforms for real time applications.

To evaluate the computational burden of the proposed OSSDPC algorithm, its execution time has been measured. Fig. 11a shows graphically the execution time of the algorithm. To do this, one of the DSP pins is set to ' 1 ' when the internal interruption stars. Then it is toggled to ' 0 ' when the algorithm is finished. It can be observed that the time needed to perform all the calculations is roughly $90 \mu \mathrm{s}$. This shows that the proposed OSS-DPC strategy requires intensive computations.

Taking into account that, in the experiment, the sampling period is $100 \mu \mathrm{s}$, it turns out that OSS-DPC uses $90 \%$ of the available time. Thus, it is not possible to increase the sampling frequency in the selected hardware platform. Consequently, the control strategy cannot take advantage of PWM-SVM optimizations including its ability to duplicate the sampling frequency and maintain the switching frequency to increase the system performance without increasing the switching losses.

In order to reduce the number of computations needed, the following observations can be made. In the $\alpha \beta$ frame $v_{r 0}=$ $v_{r 7}$. Therefore, evaluations of sectors 1 and 2 lead to the same control action value. The same conclusion can be obtained for sectors pairs 3-4, 5-6, 7-8, 9-10 and 11-12. Thus, the control algorithm only needs to be evaluated for six sectors, from A to $\mathrm{F}$, as presented in Fig. 12. 
TABLE III

PARAMETERS $f_{p i}$ AND $f_{q i}$ TO BE APPLIED WITH ROSS-DPC.

\begin{tabular}{ccccccc}
\hline \hline Sector & $f_{p 1}$ & $f_{p 2}$ & $f_{p 3}$ & $f_{q 1}$ & $f_{q 2}$ & $f_{q 3}$ \\
\hline A & $f_{p, v_{r 0}}$ & $f_{p, v_{r 1}}$ & $f_{p, v_{r 2}}$ & $f_{q, v_{r 0}}$ & $f_{q, v_{r 1}}$ & $f_{q, v_{r 2}}$ \\
B & $f_{p, v_{r 0}}$ & $f_{p, v_{r 3}}$ & $f_{p, v_{r 2}}$ & $f_{q, v_{r}}$ & $f_{q, v_{r 3}}$ & $f_{q, v_{r 2}}$ \\
C & $f_{p, v_{r 0}}$ & $f_{p, v_{r 3}}$ & $f_{p, v_{r 4}}$ & $f_{q, v_{r 0}}$ & $f_{q, v_{r 3}}$ & $f_{q, v_{r 4}}$ \\
D & $f_{p, v_{r 0}}$ & $f_{p, v_{r 5}}$ & $f_{p, v_{r 4}}$ & $f_{q, v_{r 0}}$ & $f_{q, v_{r}}$ & $f_{q, v_{r 4}}$ \\
E & $f_{p, v_{r 0}}$ & $f_{p, v_{r 5}}$ & $f_{p, v_{r 6}}$ & $f_{q, v_{r 0}}$ & $f_{q, v_{r}}$ & $f_{q, v_{r 6}}$ \\
$\mathrm{~F}$ & $f_{p, v_{r 0}}$ & $f_{p, v_{r 1}}$ & $f_{p, v_{r 6}}$ & $f_{q, v_{r 0}}$ & $f_{q, v_{r 1}}$ & $f_{q, v_{r 6}}$ \\
\hline \hline
\end{tabular}

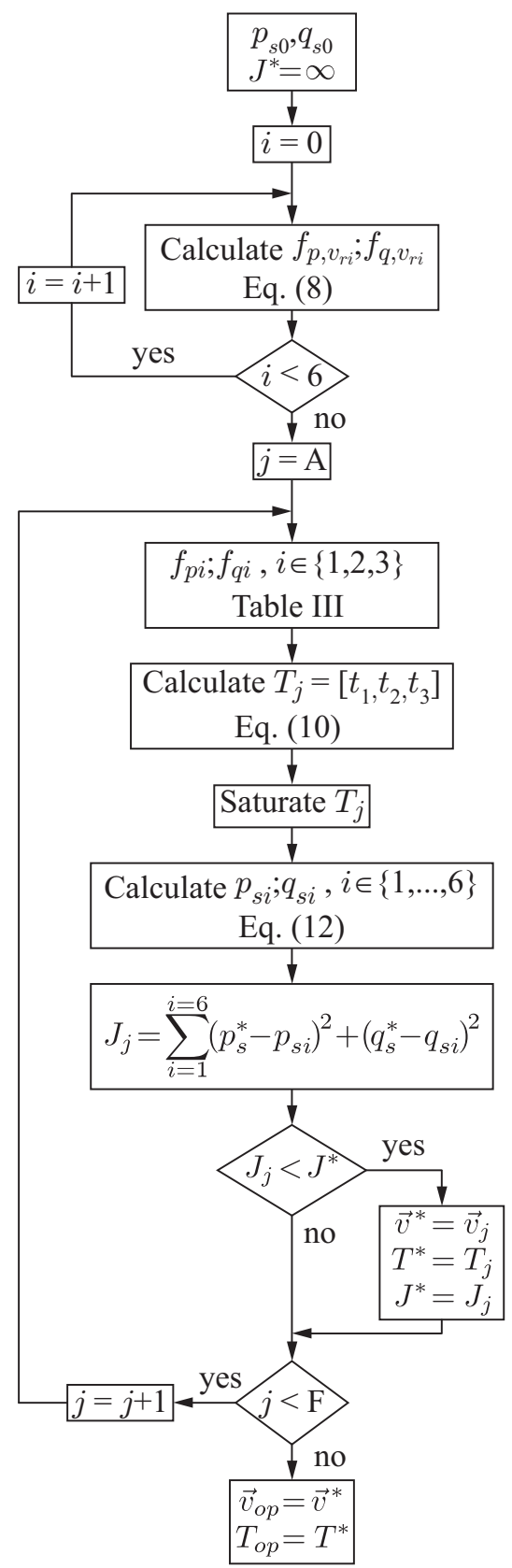

Fig. 13. Flow diagram of the proposed ROSS-DPC algorithm.

On the other hand, vector $v_{r 1}$ is common to sectors $\mathrm{A}$ and F. Similarly, $v_{r 2}$ belongs to sectors $\mathrm{A}$ and $\mathrm{B}, v_{r 3}$ to sectors $\mathrm{B}$ and $\mathrm{C}, v_{r 4}$ to sectors $\mathrm{C}$ and $\mathrm{D}, v_{r 5}$ to sectors $\mathrm{D}$ and $\mathrm{E}$, and $v_{r 6}$ to sectors $\mathrm{E}$ and $\mathrm{F}$. Further, $v_{r 0}$ and $v_{7}$ are included in

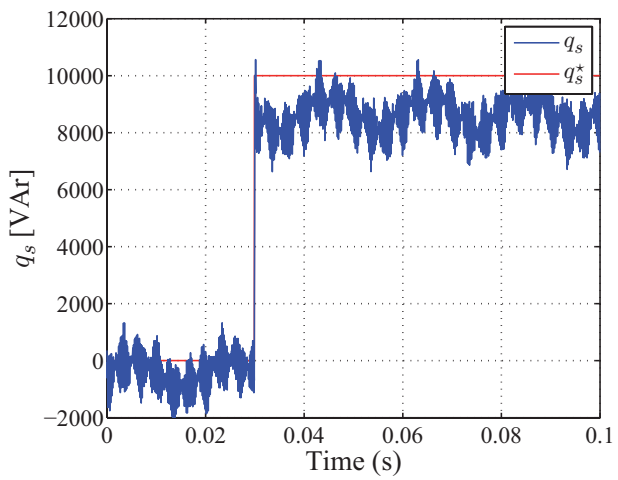

(a)

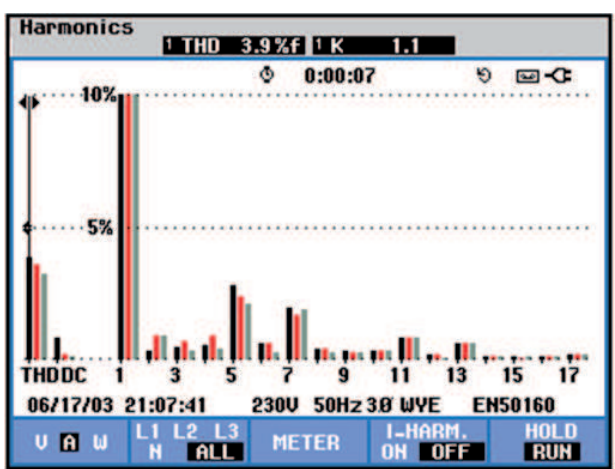

(b)

Fig. 14. Experimental results for the ROSS-DPC algorithm: (a) System response for an instantaneous reactive power command step from $0 \mathrm{kVAr}$ to $10 \mathrm{kVAr}$. (b) Harmonic content of the output currents.

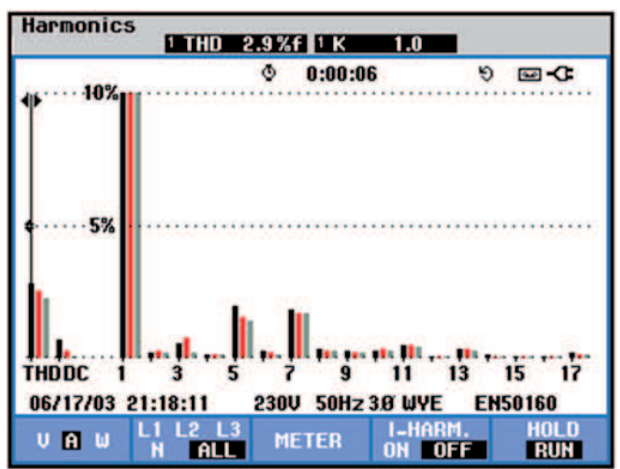

Fig. 15. Harmonic content of the output currents for the ROSS-DPC with $f_{s}$ $=20 \mathrm{kHz}$ and $f_{\text {sw }}=10 \mathrm{kHz}$.

all sectors. In this way, the values $f_{p i}$ and $f_{q i}$ for vector $v_{r i}$, denoted as $f_{p, v_{r i}}$ and $f_{q, v_{r i}}$ respectively, can be calculated in advance to the switching times corresponding to each sector, thereby providing a more efficient algorithm. Once a sector is selected, Table III can be used to choose the corresponding values of $f_{p i}$ and $f_{q i}$.

The above considerations lead to the reduced OSS-DPC (ROSS-DPC) algorithm. The flow diagram of the proposed ROSS-DPC strategy is shown in Fig. 13. The execution time of the ROSS-DPC has also been assessed graphically as presented in Fig. 11b. which demonstrate that the new algorithm only needs roughly $40 \mu$ s for computing all calculations. Therefore, the computational burden of the OSS-DPC has been reduced 


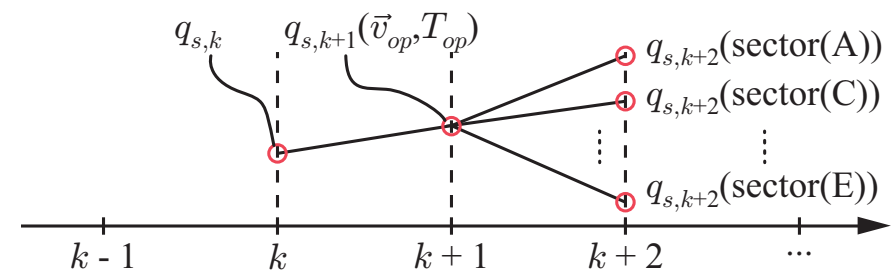

Fig. 16. Sampling delay compensation for the proposed predictive control strategy.

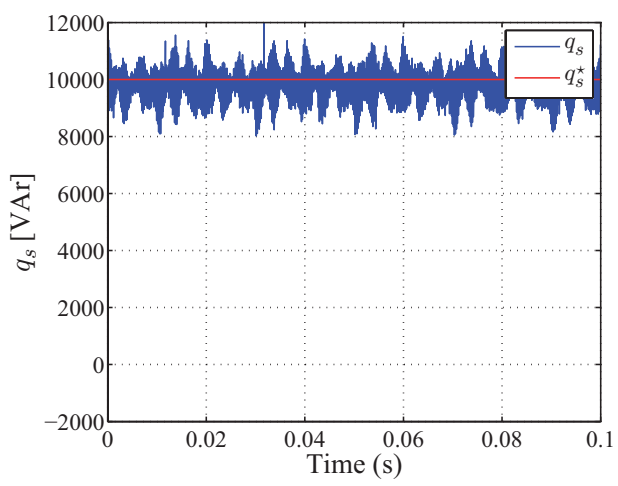

(a)

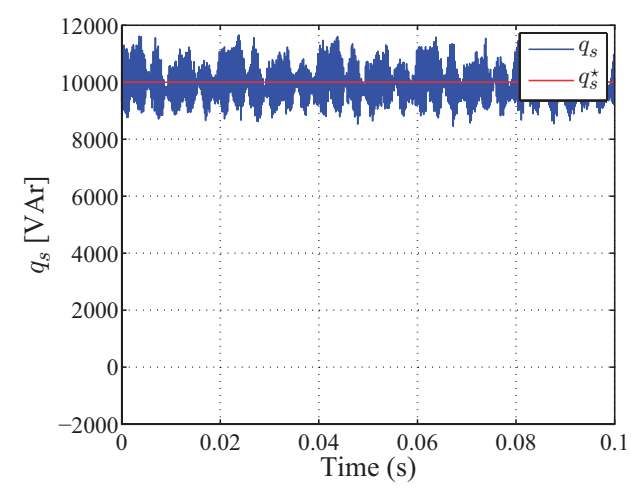

(b)

Fig. 17. Steady state response for an instantaneous reactive power command of 10 kVAr: (a) ROSS-DPC algorithm without delay compensation. (b) ROSSDPC algorithm with delay compensation.

by $55 \%$. It is important to emphasize that only the number of computations have been reduced. The performance remains the same as shown in Fig. 14. Both transient and steady state responses are equal to those exhibited by OSS-DPC.

Due to the computational savings, it is possible to run the ROSS-DPC using a sampling frequency of $f_{s}=20 \mathrm{kHz}$ and a switching frequency of $f_{s w}=10 \mathrm{kHz}$. This improves the THD of the output current without increasing the switching losses. The harmonic spectrum of the output currents is presented in Fig. 15. It should be noticed that the THD is $2.9 \%$. Thus, a reduction of about $30 \%$ has been achieved by increasing the sampling frequency.

\section{B. Delay compensation}

The implementation of the control algorithm on a digital platform introduces a delay in the control action which deteriorates the closed-loop performance of the system. This is due

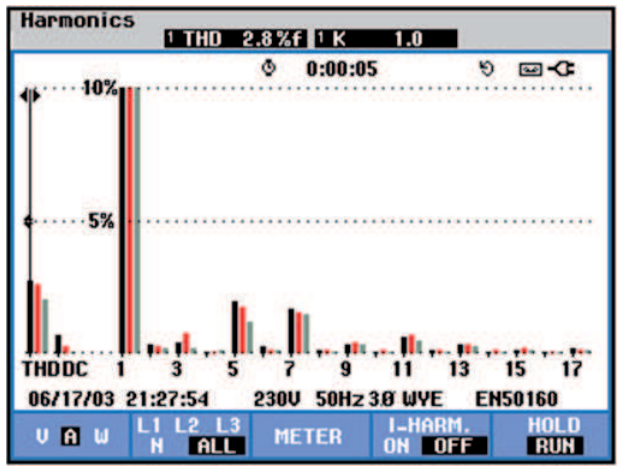

Fig. 18. Harmonic content of the output currents for the ROSS-DPC with delay compensation, $f_{s}=20 \mathrm{kHz}$ and $f_{s w}=10 \mathrm{kHz}$.

to the fact that, to account for computation times, the control action is applied one sampling period after it is calculated.

In the case of MPC strategies, this can be easily handled by considering predictions at instant $k+2$ instead of instant $k+1$, [35], [36]. This is shown in Fig. 16 for the instantaneous reactive power variable. The value of the instantaneous reactive power at instant $k\left(q_{s, k}\right)$ can be calculated from the measurements $v_{s \alpha \beta, k}$ and $i_{s \alpha \beta, k}$. The value of this variable at instant $k+1$ only depends on $v_{s \alpha \beta, k}$ and $i_{s \alpha \beta, k}$ and the switching function applied to the power converter. This switching function was calculated in the previous sampling instant by the ROSS-DPC and is defined by $\vec{v}_{o p}$ and $T_{o p}$. Therefore, it is possible to obtain a prediction of $q_{s, k+1}$. Predictions at instant $k+2$ are needed to compute the algorithm. It should be noted that $q_{s, k+2}$ depends on the sector evaluated and therefore it is necessary to calculate predictions for the values $f_{p i}$ and $f_{q i}$ at $k+1, f_{p i, k+1}$ and $f_{q i, k+1}$ respectively.

Taking into account the definitions for the instantaneous active and reactive power, $f_{p i, k+1}$ and $f_{q i, k+1}$ can be calculated by

$$
\begin{aligned}
f_{p i, k+1} & =\frac{v_{s \alpha, k+1}}{L}\left(v_{r \alpha}-v_{s \alpha, k+1}\right) \\
& +\frac{v_{s \beta, k+1}}{L}\left(v_{r \beta}-v_{s \beta, k+1}\right) \\
& -\omega q_{s, k+1} \\
f_{q i, k+1} & =\frac{v_{s \beta, k+1}}{L}\left(v_{r \alpha}-v_{s \alpha, k+1}\right) \\
& -\frac{v_{s \alpha, k+1}}{L}\left(v_{r i \beta}-v_{s \beta, k+1}\right) \\
& +\omega q_{s, k+1} .
\end{aligned}
$$

From (13) and (14), it is clear that predictions for $v_{s \alpha \beta, k+1}$ are needed. These values, under input balanced grid voltage can be easily obtained as

$$
\left[\begin{array}{c}
v_{s \alpha, k+1} \\
v_{s \beta, k+1}
\end{array}\right]=\left[\begin{array}{cc}
\cos \left(2 \pi f T_{s}\right) & -\sin \left(2 \pi f T_{s}\right) \\
\sin \left(2 \pi f T_{s}\right) & \cos \left(2 \pi f T_{s}\right)
\end{array}\right]\left[\begin{array}{l}
v_{s \alpha, k} \\
v_{s \beta, k}
\end{array}\right],
$$

where $T_{s}=1 / f_{s}$ is the sampling period.

The effect of the delay compensation on the performance of the ROSS-DPC strategy has been experimentally tested. For this purpose, the steady state response of the system for an 
instantaneous reactive power command of $10 \mathrm{kVAr}$ has been analyzed.

Fig. 17a and Fig. 17b present the steady state system response, without and with the delay compensation respectively. In both cases the controller behaves correctly. However, when the average value of $q_{s}$ is analyzed it shows that without delay compensation $q_{s, a v}$ is $9.723 \mathrm{kVAr}$ whereas $q_{s, a v}$ with delay compensation is $9.909 \mathrm{kVAr}$. Therefore, the relative error, defined as $100 *\left(q_{s}^{*}-q_{s, a v}\right) / q_{s}^{*}$, is reduced from $2.77 \%$ to $0.91 \%$. The compensation of the delay also improves the harmonic spectrum of the output current as depicted in Fig. 18. It can be noticed that the THD has been further reduced to $2.8 \%$

\section{CONCLUSiON}

Direct power control (DPC) strategies are a good alternative to control grid connected converters. Model predictive control (MPC) for power converters and drives has shown its ability to provide highly effective solutions. In this way, the predictive DPC (P-DPC) was developed in the literature in order to take advantage of both DPC and MPC features. However, there are still some issues to be addressed. In this paper, a new P-DPC controller, named optimal switching sequence DPC (OSSDPC), is proposed, solving the problem of conventional $\mathrm{P}$ DPC with the selection of the optimal sequence from the grid voltage sector information. The proposed OSS-DPC algorithm takes advantage of the discrete nature of the power converter by obtaining a local optimum for each of the six voltage sectors. Since only a finite number of local optima exist, the global optimum can be obtained in a similar fashion as in the FCS-MPC case.

Experiments have been conducted and the results show that the proposed control method performs well. A careful analysis of OSS-DPC showed that it is possible to reduce the number of calculations needed to implement the control strategy without reducing its performance. Additionally, the effect of the delay introduced due to digital implementation was also studied. Both considerations were addressed by deriving a new algorithm called reduced OSS-DPC (ROSSDPC), showing that the resulting predictive control strategy provides excellent closed-loop performance in both transient and steady states.

\section{REFERENCES}

[1] M. P. Kazmierkowski, L. G. Franquelo, J. Rodriguez, M. A. Perez, and J. I. Leon, "High-performance motor drives," IEEE Ind. Electron. Mag., vol. 5, no. 3, pp. 6-26, 2011.

[2] J. M. Carrasco, L. G. Franquelo, J. T. Bialasiewicz, E. Galvan, R. Portillo, M. M. Prats, J. I. Leon, and N. Moreno-Alfonso, "Power-electronic systems for the grid integration of renewable energy sources: A survey," IEEE Trans. Ind. Electron., vol. 53, no. 4, pp. 1002-1016, 2006.

[3] E. Romero-Cadaval, G. Spagnuolo, L. G. Franquelo, C. A. Ramos-Paja, T. Suntio, and W. M. Xiao, "Grid-connected photovoltaic generation plants: Components and operation," IEEE Ind. Electron. Mag., vol. 7, no. 3, pp. 6-20, 2013

[4] S. Vazquez, S. M. Lukic, E. Galvan, L. G. Franquelo, and J. M. Carrasco, "Energy storage systems for transport and grid applications," IEEE Trans. Ind. Electron., vol. 57, no. 12, pp. 3881-3895, 2010.

[5] M. Schweizer, T. Friedli, and J. W. Kolar, "Comparative evaluation of advanced three-phase three-level inverter/converter topologies against two-level systems," IEEE Trans. Ind. Electron., vol. 60, no. 12, pp. 5515-5527, 2013.
[6] E. Behrouzian, M. Bongiorno, and H. Z. De La Parra, "An overview of multilevel converter topologies for grid connected applications," in Power Electronics and Applications (EPE), 2013 15th European Conference on, 2013, pp. 1-10.

[7] J. I. Leon, L. G. Franquelo, B. Wu, and S. Kouro, "Introduction to the special section on modulation techniques for dc-to-ac power converters," IEEE Trans. Ind. Electron., vol. 60, no. 5, pp. 1859-1860, 2013.

[8] K. Ma and F. Blaabjerg, "Modulation methods for neutral-point-clamped wind power converter achieving loss and thermal redistribution under low-voltage ride-through," IEEE Trans. Ind. Electron., vol. 61, no. 2, pp. 835-845, 2014.

[9] S. Vazquez, J. A. Sanchez, M. R. Reyes, J. I. Leon, and J. M. Carrasco, "Adaptive vectorial filter for grid synchronization of power converters under unbalanced and/or distorted grid conditions," IEEE Trans. Ind. Electron., vol. 61, no. 3, pp. 1355-1367, 2014.

[10] F. Blaabjerg, R. Teodorescu, M. Liserre, and A. V. Timbus, "Overview of control and grid synchronization for distributed power generation systems," IEEE Trans. Ind. Electron., vol. 53, no. 5, pp. 1398-1409, 2006.

[11] M. Malinowski, M. P. Kazmierkowski, and A. M. Trzynadlowski, "A comparative study of control techniques for pwm rectifiers in ac adjustable speed drives," IEEE Trans. Power Electron., vol. 18, no. 6, pp. 1390-1396, 2003.

[12] R. Portillo, S. Vazquez, J. I. Leon, M. M. Prats, and L. G. Franquelo, "Model based adaptive direct power control for three-level npc converters," IEEE Trans. Ind. Informat., vol. 9, no. 2, pp. 1148-1157, 2013.

[13] T. Noguchi, H. Tomiki, S. Kondo, and I. Takahashi, "Direct power control of pwm converter without power-source voltage sensors," IEEE Trans. Ind. Appl., vol. 34, no. 3, pp. 473-479, 1998.

[14] M. Malinowski, M. Jasinski, and M. P. Kazmierkowski, "Simple direct power control of three-phase pwm rectifier using space-vector modulation (dpc-svm)," IEEE Trans. Ind. Electron., vol. 51, no. 2, pp. 447-454, 2004.

[15] S. Vazquez, J. A. Sanchez, J. M. Carrasco, J. I. Leon, and E. Galvan, "A model-based direct power control for three-phase power converters," IEEE Trans. Ind. Electron., vol. 55, no. 4, pp. 1647-1657, 2008.

[16] P. Cortes, M. P. Kazmierkowski, R. M. Kennel, D. E. Quevedo, and J. Rodriguez, "Predictive control in power electronics and drives," IEEE Trans. Ind. Electron., vol. 55, no. 12, pp. 4312-4324, 2008.

[17] S. Kouro, P. Cortes, R. Vargas, U. Ammann, and J. Rodriguez, "Model predictive control-a simple and powerful method to control power converters," IEEE Trans. Ind. Electron., vol. 56, no. 6, pp. 1826-1838, 2009.

[18] D. Quevedo, R. Aguilera, and T. Geyer, Advanced and Intelligent Control in Power Electronics and Drives, vol. 531 of Studies in Computational Intelligence. Springer International Publishing, 2014, ch. Predictive control in power electronics and drives: Basic concepts, theory, and methods, pp. 181-226.

[19] J. M. Maciejowski, Predictive Control with Constraints. London: Prentice Hall, 2002.

[20] P. Cortes, J. Rodriguez, P. Antoniewicz, and M. P. Kazmierkowski, "Direct power control of an AFE using predictive control," IEEE Trans. Power Electron., vol. 23, no. 5, pp. 2516-2523, 2008.

[21] D. E. Quevedo, R. P. Aguilera, M. A. Perez, P. Cortes, and R. Lizana, "Model predictive control of an AFE rectifier with dynamic references," IEEE Trans. Power Electron., vol. 27, no. 7, pp. 3128-3136, 2012.

[22] S. Aurtenechea, M. A. Rodriguez, E. Oyarbide, and J. R. Torrealday, "Predictive direct power control - a new control strategy for dc/ac converters," in IEEE 32nd Annual Conference on Industrial Electronics (IECON 2006), 2006, pp. 1661-1666.

[23] S. Vazquez, J. Leon, L. Franquelo, J. Rodriguez, H. Young, A. Marquez, and P. Zanchetta, "Model predictive control: A review of its applications in power electronics," IEEE Ind. Electron. Mag., vol. 8, no. 1, pp. 16-31, March 2014.

[24] S. A. Larrinaga, M. A. R. Vidal, E. Oyarbide, and J. R. T. Apraiz, "Predictive control strategy for dc/ac converters based on direct power control," IEEE Trans. Ind. Electron., vol. 54, no. 3, pp. 1261-1271, 2007.

[25] C. Xia, M. Wang, Z. Song, and T. Liu, "Robust model predictive current control of three-phase voltage source pwm rectifier with online disturbance observation," IEEE Trans. Ind. Informat., vol. 8, no. 3, pp. 459-471, 2012.

[26] Z. Song, C. Xia, and T. Liu, "Predictive current control of three-phase grid-connected converters with constant switching frequency for wind energy systems," IEEE Trans. Ind. Electron., vol. 60, no. 6, pp. 24512464, 2013. 
[27] J. Hu, "Improved dead-beat predictive dpc strategy of grid-connected dc-ac converters with switching loss minimization and delay compensations," IEEE Trans. Ind. Informat., vol. 9, no. 2, pp. 728-738, 2013.

[28] J. Hu and Z. Q. Zhu, "Improved voltage-vector sequences on dead-beat predictive direct power control of reversible three-phase grid-connected voltage-source converters," IEEE Trans. Power Electron., vol. 28, no. 1, pp. 254-267, 2013.

[29] Z. Song, W. Chen, and C. Xia, "Predictive direct power control for threephase grid-connected converters without sector information and voltage vector selection," IEEE Trans. Power Electron., vol. PP, no. 99, pp. 1-1, 2013.

[30] N. Kutasi, K. György, and A. Kelemen, "Constant-frequency constrained optimal direct power control of voltage-source pwm rectifiers," Acta Electrotehnica, Mediamira Science Publisher, vol. 55, no. 2, pp. 138$144,2010$.

[31] A. Kelemen, N. Kutasi, M. Imecs, and I. Incze, "Constrained optimal direct power control of voltage-source pwm rectifiers," in Intelligent Engineering Systems (INES), 2010 14th International Conference on, May 2010, pp. 249-254.

[32] R. P. Aguilera, D. E. Quevedo, S. Vazquez, and L. G. Franquelo, "Generalized predictive direct power control for ac/dc converters," in IEEE ECCE Asia Downunder (ECCE Asia 2013), 2013, pp. 1215-1220.

[33] H. Akagi, E. H. Watanabe, and M. Aredes, Instantaneous Power Theory and Applications to Power Conditioning, ser. IEEE Press Series on Power Engineering. Wiley, 2007.

[34] R. P. Aguilera, P. Lezana, and D. E. Quevedo, "Finite-control-set model predictive control with improved steady-state performance," IEEE Trans. Ind. Informat., vol. 9, no. 2, pp. 658-667, 2013.

[35] P. Lezana, R. Aguilera, and D. E. Quevedo, "Model predictive control of an asymmetric flying capacitor converter," IEEE Trans. Ind. Electron., vol. 56, no. 6, pp. 1839-1846, 2009.

[36] P. Cortes, J. Rodriguez, C. Silva, and A. Flores, "Delay compensation in model predictive current control of a three-phase inverter," IEEE Trans. Ind. Electron., vol. 59, no. 2, pp. 1323-1325, 2012.

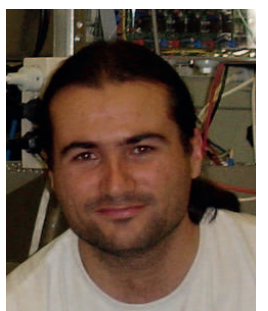

Sergio Vazquez (S'04, M'08) was born in Seville, Spain, in 1974. He received the B.S, M.S. and PhD degrees in industrial engineering from the University of Seville (US) in 2003, 2006, and 2010, respectively. In 2002, he was with the Power Electronics Group, US, working in R\&D projects. He is currently an Associate Professor with the Department of Electronic Engineering, US. His research interests include power electronics systems, modeling, modulation and control of power electronics converters applied to renewable energy technologies.

Dr. Vazquez was recipient as coauthor of the 2012 Best Paper Award of the IEEE Transactions on Industrial Electronics.

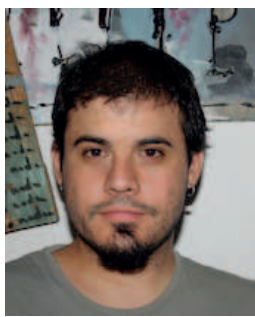

Abraham Marquez was born in Huelva, Spain, in 1985. He received his B.Eng. degree in telecommunications engineering from the University of Seville (US), Spain, in 2014. He was granted a scholarship from the Asociación de Investigación y Cooperación Industrial de Andalucía to pursue his M.S. degree in power electronics at US. His main research interest is the model-based predictive control of power converters and drives.

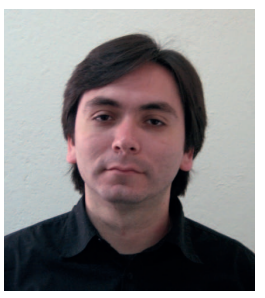

Ricardo Aguilera (S'01, M'12) Ricardo Aguilera(S'01, M'12) received his B.Sc. degree in Electrical Engineering from the Universidad de Antofagasta, Chile, 2003, M.Sc. degree in Electronics Engineering from the Technical University Federico Santa Maria (UTFSM), Chile, 2007, and Ph.D. degree in Electrical Engineering from The University of Newcastle, Australia, 2012.

From 2003 to 2004, he was a Research Assistant at the UTFSM. Then, from 2012 to 2013, he was a Research Academic at the School of Electrical Engineering and Computer Science at The University of Newcastle, Australia, where he was part of the Centre for Complex Dynamic Systems and Control. In January 2013, he joined the School of Electrical Engineering and Telecommunications at The University of New South Wales (UNSW), Sydney, Australia, where he currently holds a Senior Research Associate position at the Australian Energy Research Institute (AERI). His main research interests include power electronics, and theoretical and practical aspects on model predictive control

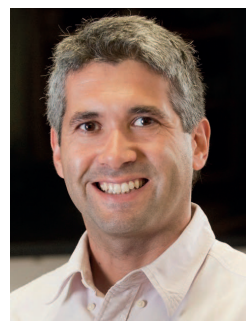

Daniel Quevedo (S'97, M'05, SM'14) received Ingeniero Civil Electronico and Magister en Ingenieria Electronica degrees from the Universidad Tecnica Federico Santa Maria, Valparaiso (UTFSM), Chile in 2000. In 2005, he received the Ph.D. degree from The University of Newcastle, Australia, where he is currently an Associate Professor. He has been a visiting researcher at various institutions, including Uppsala University, KTH Stockholm, Kyoto University, Karlsruher Institut für Technologie, University of Notre Dame, INRIA Grenoble, The Hong Kong University of Science and Technology, Aalborg University, and NTU Singapore.

Dr. Quevedo was supported by a full scholarship from the alumni association at the UTFSM and received several university-wide prizes upon graduating. He received the IEEE Conference on Decision and Control Best Student Paper Award in 2003 and was also a finalist in 2002. In 2009 he was awarded a five-year Australian Research Fellowship. His research interests include automatic control, signal processing, and power electronics.

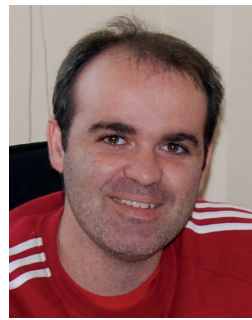

Jose I. Leon (S'04, M'07, SM'14) was born in Cadiz, Spain, in 1976. He received the B.S., M.S., and Ph.D. degrees in telecommunications engineering from the University of Seville (US), Seville, Spain, in 1999, 2001, and 2006, respectively. He is currently an Associate Professor with the Department of Electronic Engineering, US. His research interests include electronic power systems, modulation and control of power converters, and renewable energy systems.

Dr. Leon was recipient as coauthor of the 2008 Best Paper Award of the IEEE Industrial Electronics Magazine and the 2012 Best Paper Award of the IEEE Transactions on Industrial Electronics. He was recipient of the 2014 IEEE IES Early Career Award and is currently serving as Associated Editor of the IEEE Transactions on Industrial Electronics.

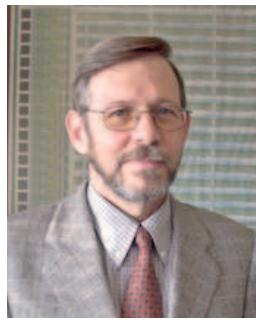

Leopoldo G. Franquelo (M'84, SM'96, F'05) He received the M.Sc. and Ph.D. degrees in electrical engineering from the University de Seville (US), Spain, in 1977 and 1980, respectively. He is currently with the Department of Electronics Engineering, University of Seville. His current research interests include modulation techniques for multilevel inverters and its application to power electronic systems for renewable energy systems.

Dr. Franquelo has been a Distinguished Lecture since 2006 and an Associated Editor for the IEEE TRANSACTIONS ON INDUSTRIAL ELECTRONICS since 2007 and coEiC since 2014. Member at large of the IES AdCom (2002-03), the VicePresident for Conferences (2004-07), and the President Elect of the IES (200809). He was the President of the IEEE Industrial Electronics Society (201011). 